Las Torres de Lucca. Revista internacional de filosofía política

ISSN-e: 2255-3827

\title{
Epistemología de la Democracia ${ }^{1}$
}

Elizabeth Anderson ${ }^{2}$

Recibido: 10-11-2020 / Aceptado: 15-5-2021/ Publicado: 30-01-2022

Resumen: Este trabajo investiga las capacidades epistémicas de las instituciones democráticas a través de una evaluación de tres modelos epistémicos de democracia: el Teorema del Jurado de Condorcet, el Teorema 'Diversidad supera Habilidad' y el modelo experimentalista de Dewey. El modelo de Dewey es superior a los demás en su capacidad de modelar las funciones epistémicas de tres características constitutivas de la democracia: la diversidad epistémica de los participantes, la interacción de la votación con el debate y los mecanismos de retroalimentación como las elecciones periódicas y las protestas. Considera que la democracia es una institución para reunir información ampliamente distribuida sobre problemas y políticas de interés público mediante la participación de conocedores epistémicamente diversos. Las normas democráticas de libre discurso, disenso, retroalimentación y rendición de cuentas funcionan para garantizar el aprendizaje colectivo y con base experimental a partir de las diversas experiencias de los diferentes conocedores. Ilustro estos puntos con un estudio de caso de grupos forestales comunitarios en el Asia meridional, cuyas capacidades epistémicas se han visto perjudicadas por la supresión de la participación de las mujeres.

Palabras claves: disenso; diversidad; epistemología institucional; falibilismo; Dewey; Condorcet.

\section{[en] The Epistemology of Democracy}

Abstract: This paper investigates the epistemic powers of democratic institutions through an assessment of three epistemic models of democracy: the Condorcet Jury Theorem, the Diversity Trumps Ability Theorem, and Dewey's experimentalist model. Dewey's model is superior to the others in its ability to model the epistemic functions of three constitutive features of democracy: the epistemic diversity of participants, the interaction of voting with discussion, and feedback mechanisms such as periodic elections and protests. It views democracy as an institution for pooling widely distributed information about problems and policies of public interest by engaging the participation of epistemically diverse knowers. Democratic norms of free discourse, dissent, feedback, and accountability function to ensure collective, experimentally-based learning from the diverse experiences of different knowers. I illustrate these points with a case study of community forestry groups in South Asia, whose epistemic powers have been hobbled by their suppression of women's participation.

Keywords: dissent; diversity; institutional epistemology; falibilism; Dewey; Condorcet.

Cómo citar: Anderson, E. (2022). Epistemología de la Democracia. Las Torres de Lucca. Revista internacional de filosofía política, 11(1), 117-127. https://dx.doi.org/10.5209/ltdl.79873

\section{Nota de lxs traductorxs}

Agradecemos a la autora por el permiso concedido para la traducción de este artículo, así como también por cubrir los derechos de la publicación.

Traducción: Blas Radi, IIF/SADAF, CONICET, Universidad de Buenos Aires

Correo electrónico: blasradi@filo.uba.ar

ORCID: https://orcid.org/0000-0002-1990-3600

Revisión: Julia Rabanal, Universidad de Buenos Aires

Correo electrónico: juliadiaz2@hotmail.com

ORCID: https://orcid.org/0000-0002-8823-7410

Una rama importante de la epistemología social investiga las capacidades epistémicas de las instituciones.

1 El artículo fue publicado originalmente en la revista Episteme, 3(1-2), 8-22 doi:10.3366/epi.2006.3.1-2.8

Agradezco a Bina Agarwal, David Estlund, Scott Page, Joseph Rouse, Alison Wylie y a los participantes en la Tercera Conferencia Anual de EPIS-

TEME por la útil comunicación en relación con este artículo.

2 Elizabeth Anderson, John Rawls Collegiate Professor of Philosophy and Women's Studies at the University of Michigan, Ann Arbor.

Las Torres de Lucca. 11 (1), 2022: 117-127 
Llamemos a esta rama "epistemología institucional." Ella hace preguntas como esta: ¿tienen las instituciones de un tipo particular la capacidad de reunir y utilizar eficazmente la información que necesitan para resolver un problema particular? Dadas las capacidades epistémicas de tales instituciones, ¿qué problemas deberían asignárseles? ¿Cómo pueden diseñarse para mejorar sus capacidades epistémicas?

Estas cuestiones son de particular importancia cuando los problemas que debemos resolver exigen la utilización de información que se encuentra sumamente dispersa en la sociedad. La información que está en la mente de muchos actores dispares debe ser incorporada de alguna manera en la solución del problema. Las instituciones pueden evaluarse según su capacidad de movilizar y responder a la información requerida. Friedrich Hayek (1945), un teórico pionero en epistemología institucional, estableció una de sus conclusiones centrales: que el problema de la asignación eficiente de recursos no puede resolverse mediante una planificación estatal centralizada porque ningún órgano central es capaz de reunir en sí mismo toda la información ampliamente dispersa que se necesita para resolver este problema. El único vehículo adecuado para transmitir la información requerida son los precios de mercado. Los mercados generan y transmiten de una forma única la información necesaria; lxs planificadorxs centrales no tienen acceso a ella de manera independiente del mercado. Por lo tanto, el problema de la asignación de recursos debe asignarse a los mercados, no a los Estados.

La información socialmente dispersa puede transmitirse de tres formas: el discurso (talk), los votos y los precios de mercado. Los mercados responden principalmente a la información sobre los precios; los estados democráticos, principalmente al discurso y los votos. Así que el trabajo de Hayek plantea la cuestión de qué problemas necesitamos que sean resueltos mediante los votos y el discurso -las instituciones democráticas-. Más allá de esta cuestión, la epistemología institucional exige una evaluación de las capacidades epistémicas de los diseños alternativos de las instituciones democráticas. En este trabajo, propondré un análisis epistémico de las instituciones democráticas diseñado para avanzar en este objetivo. Mi primera tarea será construir un modelo de democracia que represente adecuadamente sus capacidades epistémicas y revele las funciones epistémicas de sus instituciones constitutivas (tales como las elecciones periódicas y una prensa libre). Argumentaré que el enfoque experimentalista de la democracia propuesto por John Dewey ofrece un modelo de la epistemología de la democracia superior a otras alternativas. Una de las ventajas del modelo de Dewey es que nos permite representar el disenso, incluso después de que se haya tomado una decisión, como algo epistémicamente productivo, no simplemente una cuestión de error. Siguiendo el modelo de Dewey, haré un recuento de los múltiples roles epistémicos del disenso en los diferentes puntos de la toma de decisiones democrática. Por último, abordaré cuestiones de diseño democrático mediante un estudio de caso de grupos de cultivo comunitario de bosques en el sur de Asia. Estos grupos tienen como objetivo la gestión del patrimonio forestal común, a fin de proporcionar niveles sostenibles de productos forestales para uso comunitario. Mostraré cómo la exclusión de las mujeres de la participación en los grupos forestales comunitarios coarta las capacidades epistémicas de estos grupos al excluir los conocimientos situados que las mujeres tienen de las capacidades de los bosques locales. Un análisis epistémico deweyano de la democracia proporciona así un poderoso instrumento para avanzar en la reforma de las instituciones democráticas.

Las necesidades y capacidades epistémicas de cualquier institución deben ser evaluadas en relación con los problemas que necesita resolver. Comencemos, por lo tanto, con un esbozo de las características de los problemas que los estados democráticos necesitan resolver. Se trata de problemas a) de interés público, cuya solución eficaz requiere b) la acción conjunta de la ciudadanía, c) a través de la ley. Las dos últimas condiciones indican por qué la solución no puede dejarse en manos de las elecciones voluntarias no reguladas de lxs individuxs o de las asociaciones privadas. La primera establece una limitación a los problemas que pueden asignarse legítimamente a la acción del Estado. Supongamos que preguntamos qué se necesitaría para resolver el "problema" del pluralismo religioso. Facultar a la mayoría para que establezcan su religión preferida y prohíban a todos los rivales por ley resolvería este "problema" y es probablemente la única forma efectiva de resolverlo. Por lo tanto, se cumplen las dos últimas condiciones para suponer que el Estado es quien debe resolver el problema. Sin embargo, ¿para quién es esto un problema? Lxs evangelistas consideran que el hecho de que millones de personas no sean cristianas es un problema para ellxs. Pero no es un problema de interés público. Lxs ciudadanxs de las democracias modernas se han negado a autorizar al Estado a dictar a lxs individuxs cuál debe ser su religión, y por lo tanto declararon que los problemas religiosos son de interés privado. Dado que los Estados democráticos no deberían ocuparse de resolver tales problemas, sus necesidades epistémicas, por lo tanto, se reducen: los Estados no necesitan tener experticia en teología.

El requisito de que los problemas que deben resolver las instituciones democráticas sean de interés público, ayuda a explicar por qué los votos y el discurso, más que los precios, son la forma adecuada de información a la que los Estados deben responder. Los precios transmiten información sobre las preferencias privadas. Pero como hemos visto en el caso religioso anterior, el mero hecho de que una preferencia privada esté ampliamente extendida no la convierte en un interés público. El discurso es necesario para articular propuestas que hagan de ciertas preocupaciones un asunto de interés público; los votos se necesitan para ratificar esas propuestas.

Esta exposición de los problemas que deben resolver los Estados democráticos nos ayuda a elaborar criterios de éxito para las instituciones democráticas. Existe una tensión de larga data en la teoría democrática entre los relatos de éxito que son internos y externos al proceso democrático de toma de decisiones (Estlund, 1997). Lxs 
internistas o procedimentalistas sostienen que para reivindicar un proceso de toma de decisiones solo hay que mostrar que es justo desde el punto de vista procedimental. Esta posición descuida las funciones instrumentales de la democracia. Si decidimos que un problema, como la contaminación del aire, es de interés público y que su solución requiere una acción conjunta en el marco de la ley, no nos limitamos a tirar una moneda para decidir qué leyes sobre contaminación promulgar, aunque esto sea justo desde el punto de vista procedimental. Más bien, juzgaremos el éxito de las instituciones democráticas según criterios que son (parcialmente) externos al proceso de toma de decisiones: ¿las leyes de contaminación promulgadas reducen realmente la contaminación a niveles aceptables y a un costo aceptable? La popularidad ex ante de una ley -su aprobación por mayoríapuede hacer que su promulgación sea legítima. Pero eso no garantiza que la ley tenga éxito. El que la ley logre resolver el problema para el que fue redactada depende de sus consecuencias externas y no, o no simplemente, de la equidad del procedimiento por el que fue promulgada.

Así pues, al menos parte del criterio de éxito de las leyes es externo al proceso de toma de decisiones. Las leyes pueden hacer las cosas bien o mal. Lxs demócratas epistémicxs se centran en la cuestión de si se puede confiar en las instituciones democráticas para tomar las decisiones correctas según criterios externos. Sin embargo, no podemos juzgar el éxito de la ley solo por criterios externos e instrumentales. Que un problema cuente o no como de interés público genuino se determina en parte por si es un objeto real de preocupación pública -es decir, por el hecho de que lxs ciudadanxs o sus representantes afirmen su lugar en la agenda pública mediante procesos de toma de decisiones equitativos desde el punto de vista procedimental-. Además, el hecho de que la solución funcione depende de qué consecuencias (posiblemente no deseadas) el público considere aceptables, lo que a su vez está parcialmente determinado por los procesos democráticos de toma de decisiones. Por lo tanto, los criterios de éxito de las instituciones democráticas son en parte internos y en parte externos al proceso de toma de decisiones (Richardson, 1997).

\section{Tres modelos epistémicos de democracia}

Con este esbozo del tipo de problema que la democracia necesita resolver y de los criterios internos y externos de éxito para sus soluciones, podemos ahora pasar a la cuestión de cómo modelar los poderes de la democracia para reunir y desplegar la información necesaria para elaborar soluciones sólidas a sus problemas. Tres modelos ofrecen análisis epistémicos de la democracia: el Teorema del Jurado de Condorcet, el Teorema de 'Diversidad supera Habilidad', y el enfoque experimentalista de Dewey sobre la democracia. Evaluaré estos modelos según los siguientes criterios: a) ¿Exhiben las funciones epistémicas de las instituciones constitutivas de la democracia? b) ¿revelan los méritos y deméritos epistémicos de esas instituciones? y c) ¿proporcionan directrices para mejorar sus capacidades epistémicas? Argumentaré que, de los tres, el de Dewey ofrece el mejor modelo de las capacidades epistémicas de las instituciones democráticas y las formas de mejorar su éxito epistémico.

El enfoque epistémico más popular de la democracia se basa en el Teorema del Jurado de Condorcet (1785/1995). Este teorema establece que si lxs votantes a) se enfrentan a dos opciones, b) votan independientemente entre sí, c) votan según su juicio sobre cuál debería ser la solución correcta del problema (es decir, no votan estratégicamente), y d) tienen, en promedio, una probabilidad superior al $50 \%$ de estar en lo cierto, entonces, a medida que el número de votantes se acerca al infinito, la probabilidad de que el voto de la mayoría dé la respuesta correcta se aproxima a 1 (y se acerca rápidamente a 1 incluso con un número modesto de votantes). Dada la fuerza de este resultado, no es de extrañar que muchxs demócratas epistémicxs hayan defendido el Teorema del Jurado de Condorcet como la clave para reivindicar las capacidades epistémicas de la democracia (Cohen, 1986; Gaus, 1997; Grofman y Feld, 1988). Los resultados favorables del Teorema del Jurado de Condorcet se han generalizado para abarcar la votación plural entre múltiples opciones (Lista, 2001), las reglas de votación por supermayoría (Fey, 2003), e incluso algunos casos en los que lxs votantes individuales tienen menos del 50\% de posibilidades de tener razón (Estlund, 1997, 188).

A pesar de estos alentadores resultados, el Teorema del Jurado de Condorcet no ofrece un enfoque particularmente esclarecedor de las capacidades epistémicas de la democracia. En primer lugar, funciona si lxs votantes son epistémicamente homogénexs (de hecho, la prueba original de Condorcet suponía homogeneidad). Sin embargo, una parte importante del argumento epistémico a favor de la democracia descansa en la diversidad epistémica de lxs votantes. La mayoría de los problemas que se espera que las democracias resuelvan son complejos, y tienen efectos distribuidos asimétricamente entre individuxs según su ubicación geográfica, clase social, ocupación, educación, género, edad, raza, etc. Dado que 1xs individuxs son quienes están más familiarizadxs con los efectos que los problemas y políticas tienen sobre ellxs mismxs y las personas cercanas, la información sobre estos efectos también está distribuida asimétricamente. Seguramente una parte importante del argumento a favor de los méritos epistémicos de la democracia se basa en su capacidad de reunir esta información distribuida de manera asimétrica sobre los efectos de los problemas y las políticas, a fin de idear soluciones que respondan a las preocupaciones de todos. Por lo tanto, necesitamos un modelo de democracia 
en el que el éxito epistémico sea un producto de su capacidad para aprovechar la diversidad epistémica de lxs individuxs.

En segundo lugar, el Teorema del Jurado de Condorcet supone que lxs votantes votan independientemente unxs de otrxs. Aunque esto no excluye toda influencia de lxs votantes entre sí (Estlund, 1994), no está claro si el Teorema es robusto bajo los patrones reales de influencia característicos de las democracias modernas (Estlund, 1997, p. 189). Lo que es más importante, la libertad de prensa, el debate público y, por tanto, la influencia mutua antes de la votación son características constitutivas, no accidentales, de la democracia. Sin acceso a los foros públicos para compartir información y opiniones más allá de su conocimiento inmediato, lxs votantes están desinformados y a menudo desamparadxs (considérese la vacuidad del voto de lxs accionistas, dado que pocxs de ellxs tienen información sobre los registros y cargos de las personas nominadas a los consejos de administración de las empresas). El Teorema del Jurado de Condorcet coloca a las dos formas de reunir información características de la democracia -el voto y el discurso-potencialmente en desacuerdo entre sí. Un modelo adecuado debería mostrar cómo trabajan juntas. Antes de la votación se requiere de discusión, en parte para ayudar a lxs votantes a determinar qué problemas son realmente de interés público. Sin esa discusión, no tienen mucho por lo que guiarse más allá de sus preferencias privadas. Pero las preferencias privadas no blanqueadas no son el mejor aporte a la toma de decisiones democráticas (Anderson, 2002; Herzog, 2000), precisamente porque, como hemos visto en el caso de la religión, no constituyen un interés público, ni siquiera en conjunto. Por lo tanto, además de que no logra modelar las funciones epistémicas de las prácticas democráticas fundamentales, el Teorema del Jurado de Condorcet también potencialmente enfrenta entre sí a los criterios internos y externos de éxito de la democracia.

Tercero, el Teorema del Jurado de Condorcet no logra captar las características dinámicas de las funciones epistémicas de la democracia. El hecho de que las leyes logren resolver los problemas públicos para los que fueron diseñadas es una función de sus consecuencias, no de su popularidad ex ante. A menudo, las mayorías convergen en una solución ineficaz porque no logran anticipar ciertas consecuencias de las políticas que adoptan. Cuando se promulgó la Parte D de Medicare, que proporcionaba cobertura de recetas para lxs ciudadanxs de edad avanzada de los Estados Unidos, la mayoría pensó que la falta de dinero era el principal obstáculo para el acceso efectivo de las personas mayores a los medicamentos necesarios. Pocxs previeron que la proliferación de planes de seguros privados subvencionados, cada uno con diferentes normas y formularios, causaría confusión entre las personas mayores, especialmente en conjunción con el aumento de las tasas de demencia, o que esto también aumentaría drásticamente los costos administrativos de lxs proveedorxs de atención de la salud, como los asilos de ancianxs. La toma democrática de decisiones debe reconocer su propia falibilidad y, por consiguiente, debe instituir mecanismos de retroalimentación que le permitan aprender a idear mejores soluciones y corregir su rumbo a la luz de la nueva información sobre las consecuencias de sus políticas. Las elecciones periódicas son un mecanismo clave de retroalimentación de este tipo. El Teorema del Jurado de Condorcet no representa la necesidad de tales mecanismos. Dado que sugiere que las mayorías son casi infalibles desde el principio, ¿por qué tendrían que corregir su decisión inicial? La simplicidad de la representación de la democracia que brinda el Teorema del Jurado de Condorcet, al representar solo el momento de la votación, también impide investigar cómo mejorar el funcionamiento epistémico de las instituciones democráticas más allá del cuarto de votación.

El Teorema 'Diversidad supera Habilidad' ayuda a resolver algunas de las deficiencias del Teorema del Jurado de Condorcet. Este teorema establece que si a) el problema es difícil (ningún individux lo resuelve bien siempre), b) las personas encargadas de resolver el problema convergen en un conjunto finito de soluciones, c) las personas encargadas de resolver el problema son epistémicamente diversas (no todas convergen en el mismo óptimo local) y d) hay muchas de estas personas que trabajan juntas en grupos de tamaño moderado, entonces un conjunto de personas encargadas de resolver el problema seleccionadas al azar supera al conjunto de las mejores entre ellas (Hong y Page, 2004; Page, 2007). Surowiecki (2004) ofrece un apoyo adicional a la intuición general que subyace al teorema DSH: que conjuntos diversos de no expertxs hacen un mejor trabajo que lxs expertxs en la resolución de muchos problemas. El Teorema DSH apoya la afirmación de que la democracia, que permite que todxs participen en la solución colectiva de problemas, es epistémicamente superior a la tecnocracia o al gobierno de lxs expertxs.

El teorema DSH, aunque inicialmente fue construido para modelar la resolución de problemas en empresas, representa muchas de las características epistémicamente relevantes de la toma de decisiones democráticas que son descuidadas por el Teorema del Jurado de Condorcet. En primer lugar, el teorema DSH representa explícitamente la diversidad epistémica de los aportes de lxs ciudadanxs a la toma de decisiones democráticas como una ventaja epistémica. Segundo, el teorema DSH modela algunas de las funciones epistémicas de las asociaciones de ciudadanxs y los partidos políticos. Los partidos organizan las múltiples preocupaciones de lxs ciudadanxs en cuestiones, agendas y plataformas, y de ese modo afinan la lista de soluciones disponibles a un número manejable. También ayudan a lxs diversxs ciudadanxs a trabajar juntxs en grupos más pequeños para elaborar propuestas de solución a los problemas. En tercer lugar, el teorema DSH modela la discusión como epistémicamente productiva, no meramente como algo que potencialmente interfiere con las virtudes epistémicas de la agregación de votos. Por último, se centra en los tipos de problemas en cuya solución 
esperaríamos que las democracias fueran superiores -es decir, problemas complejos-. Es plausible suponer que los gobiernos autocráticos pueden resolver problemas relativamente sencillos, como atrapar a lxs asesinxs, tan bien como los gobiernos democráticos. Esperaríamos que los gobiernos autocráticos se desempeñen peor que los gobiernos democráticos cuando los problemas y sus soluciones son complejos, con efectos distribuidos asimétricamente y, por lo tanto, con información sobre esos efectos distribuida asimétricamente también. Lxs tomadorxs de decisiones estatales no pueden responder a diversos efectos de los que no son conscientes. Para que los Estados respondan a esos efectos se necesita el aporte de diversxs ciudadanxs, junto con un mecanismo de rendición de cuentas que garantice que esos aportes se tomen en serio. Las democracias están concebidas para responder a esos aportes; las autocracias generalmente no.

A pesar de estas virtudes del Teorema DSH, éste no logra modelar otras dos características de la democracia: la importancia no instrumental de la inclusión universal (es decir, el sufragio universal y la libertad de expresión para todxs), y los aspectos dinámicos de la democracia. El Teorema DSH representa a la diversidad epistémica como valiosa desde el punto de vista instrumental y a la inclusión universal como potencialmente valiosa, en la medida en que garantiza la inclusión de cualquier característica epistémica (información, heurística, representación perspicua) que pueda tener una clave vital para la solución de algún problema particular. Pero la inclusión universal es también esencial para garantizar la satisfacción del criterio interno de éxito para la toma democrática de decisiones: que las decisiones representen equitativamente las preocupaciones de todxs y, por lo tanto, representen un objeto de interés público. Aún más importante desde el punto de vista epistémico, el Teorema DSH no modela las funciones epistémicas de las elecciones periódicas y otros mecanismos de retroalimentación diseñados para cambiar el curso de las decisiones colectivas a la luz de la información sobre sus consecuencias.

John Dewey ofreció un enfoque experimentalista de las capacidades epistémicas de la democracia. Caracterizó a la democracia como el uso de la inteligencia social para resolver problemas de interés práctico (Dewey, 1981a; Putnam, 1990). La inteligencia práctica encarna un método experimental (Dewey, 1976). La deliberación es una especie de experimento mental en el que ensayamos soluciones posibles a determinados problemas en nuestra imaginación tratando de prever las consecuencias de su aplicación, incluyendo nuestras reacciones favorables o desfavorables a ellas. Luego ponemos a prueba las políticas que decidimos, actuando de acuerdo con ellas y evaluando los resultados. Los resultados desfavorables -cuando no se soluciona el problema para el que se adoptó la política, o cuando la solución es a costa de generar problemas peores- deben ser tratados con espíritu científico como una desconfirmación de nuestras políticas. Ellos nos dan razones para revisar nuestras políticas para que resuelvan mejor nuestros problemas. La inteligencia práctica, entonces, es la aplicación del método científico a los problemas prácticos. Esto requiere abandonar el dogmatismo, afirmar el falibilismo y aceptar las consecuencias observadas de nuestras prácticas como la evidencia clave que nos impulsa a revisarlas. Dewey consideraba que la toma democrática de decisiones era el ejercicio conjunto de la inteligencia práctica por parte de lxs ciudadanxs en general, en interacción con sus representantes y otrxs funcionarixs del Estado. Es una experimentación social cooperativa.

El modelo de Dewey es el único de los tres que representa las capacidades epistémicas de las tres características constitutivas de la democracia: diversidad, discusión y dinamismo (retroalimentación). Dewey subrayó la importancia central para la democracia de reunir a ciudadanxs de diferentes ámbitos para definir, a través de la discusión, lo que consideran problemas de interés público, y para considerar las soluciones propuestas (Dewey, 1981c). Vio que la inclusión universal de ciudadanxs diversxs era esencial para satisfacer los criterios internos y externos de éxito en la toma de decisiones democráticas. La exclusión pone en duda la afirmación de que los problemas y las soluciones, tal como los definen quienes pueden participar, son verdaderamente de interés público - que responden de manera justa a las preocupaciones de todxs, en la medida en que reivindican legítimamente la acción pública-. También socava la capacidad de la toma colectiva de decisiones para aprovechar el conocimiento situado de lxs ciudadanxs -el hecho de que ciudadanxs de diferentes ámbitos de la vida tengan diferentes experiencias de problemas y políticas de interés público-, experiencias que tienen importancia evidencial para concebir y evaluar soluciones. La inclusión universal aprovecha al máximo ese conocimiento situado que, como hemos visto, es fundamental para resolver los tipos de problemas complejos a los que se enfrentan las democracias modernas. La discusión y deliberación colectivas y democráticas son un medio para reunir esta información, distribuida asimétricamente, para la toma de decisiones.

Lo más importante es que el modelo experimentalista de democracia de Dewey nos ayuda a ver la importancia epistémica de varias instituciones democráticas que sostienen su dinamismo, su capacidad de cambio: elecciones periódicas, una prensa libre escéptica del poder del Estado, peticiones al gobierno, encuestas de opinión pública, protestas, comentarios públicos sobre los reglamentos propuestos por los organismos administrativos. En el modelo de Dewey, se trata de mecanismos de retroalimentación y rendición de cuentas que funcionan para institucionalizar el falibilismo y una actitud experimental con respecto a las políticas estatales. Empujan a los gobiernos a revisar sus políticas a la luz de la evidencia -reclamos públicos, expresados tanto en las votaciones como en los debates- de que no funcionan, o que se espera que no funcionen. En el modelo de Dewey, los votos y el discurso se refuerzan mutuamente: los votos ayudan a asegurar que lxs funcionarixs del gobierno se tomen en serio la retroalimentación verbal de lxs ciudadanxs, el discurso ayuda a definir y articular el mensaje que transmiten los votos. 
Dewey subrayaba que para que la democracia funcione no basta con instituir arreglos legales como la representación y las elecciones periódicas. La cultura también tiene que cambiar, de modo que lxs ciudadanxs en general, al interactuar entre sí en la sociedad civil, acojan con agrado la diversidad y el debate y adopten una actitud experimental respecto de los acuerdos sociales. "El futuro de la democracia se alía con la difusión de la actitud científica" hacia los asuntos prácticos (Dewey, 1981b, p. 167). Si el pueblo es escurridizo y dogmático y piensa, por ejemplo, que los arreglos sociales deben seguir la tradición o volver a los principios establecidos en un texto religioso antiguo, no estará preparado para tomar las consecuencias adversas de los hábitos actuales o de las políticas que siguen los principios antiguos como evidencia que desmiente su pretensión de éxito práctico.

El modelo experimentalista de Dewey permite una evaluación bastante fina de las capacidades epistémicas de los arreglos sociales, tanto legales como culturales. La diversidad y el debate deben encarnarse y facilitarse en las instituciones y costumbres de la sociedad civil. Si un arreglo social tiene un impacto sistemático y significativo en algún grupo social, es necesario transmitir información sobre ese impacto a las personas a cargo de la toma de decisiones. Ello requiere típicamente que el grupo se organice en una asociación o partido, de modo que sus miembros puedan compartir sus experiencias y, mediante el debate, articular las quejas compartidas y presentar propuestas para abordarlas. La falta de esas asociaciones en la sociedad civil hace que el Estado sea ciego a los efectos de sus políticas, y que las personas a cargo de la toma de decisiones sean inmunes a la rendición de cuentas por esos efectos, incluso si existe el aparato formal de la democracia. Esto ayuda a explicar por qué lxs demócratas de la Europa poscomunista han centrado tanta energía en la construcción de la sociedad civil, en lugar de en las disposiciones constitucionales: tuvieron que superar el legado del totalitarismo, que destruyó sistemáticamente las asociaciones independientes de ciudadanxs al prohibir los partidos políticos independientes y las asambleas de ciudadanxs.

Una vez que lxs ciudadanxs se organizan en organizaciones múltiples y transversales, necesitan tener acceso a canales de comunicación entre ellxs y con lxs responsables de las decisiones gubernamentales. Esto requiere que los medios de comunicación sean abiertos y accesibles a todxs. La concentración de los medios de comunicación, especialmente si permite a los titulares de cargos públicos o a un puñado de propietarixs de medios de comunicación privados censurar eficazmente la disidencia, socava las capacidades epistémicas de la democracia (un ejemplo de ello es la Italia de Silvio Berlusconi, que es propietario de la mayoría de los medios de comunicación privados de Italia y, como primer ministro, también controlaba los medios de comunicación públicos, utilizando su poder para reducir al mínimo el acceso de sus críticxs a grandes audiencias). Además, la comunicación eficaz de las quejas y propuestas requiere no solo que las personas sean libres de decir lo que piensan, sino que estén abiertas a escuchar a los demás. Si la gente difama, calla o insulta a quienes están en desacuerdo o considera la diversidad como una amenaza, las palabras de lxs excluidxs, si se atreven a hablar, caerán en oídos sordos.

La intolerancia, el abuso, el insulto por diferencias de opinión sobre religión o política o negocios, así como por diferencias de raza, color, riqueza o grado de cultura son traiciones al modo de vida democrático. Porque todo lo que impide la libertad y la plenitud de comunicación establece barreras que dividen a los seres humanos en grupos y camarillas, en sectas y facciones antagónicas y por lo tanto socava el modo de vida democrático. Las garantías meramente legales de las libertades civiles de libre creencia, libre expresión y libre reunión sirven de poco si en la vida cotidiana la libertad de comunicación, el dar y recibir ideas, hechos, experiencias, se ve ahogada por la sospecha mutua, por el abuso, por el miedo y el odio. (Dewey, 1981a, pp. 227-228).

Para concretar las capacidades epistémicas de la democracia, lxs ciudadanxs deben seguir normas que acojan o al menos toleren la diversidad y el disenso, que reconozcan la igualdad de lxs participantes en el debate concediendo a todos una escucha respetuosa, independientemente de su condición social, y que instituyan la deliberación y el dar razones, en lugar de las amenazas e insultos, como base de su comunicación con los demás. Un análisis epistémico de la democracia nos ayuda a ver que no es solo una cuestión de acuerdos legales. Es un modo de vida regido por normas culturales de igualdad, discusión y tolerancia de la diversidad.

\section{La importancia epistémica del disenso}

La diversidad y el disenso son características centrales de la democracia. Un modelo epistémico adecuado de democracia debe representar sus funciones en todas las etapas de la toma de decisiones: durante la deliberación, en el punto de decisión (votación) y después de que se haya tomado una decisión. El enfoque experimentalista de Dewey sobre la democracia es el único entre los tres modelos examinados aquí que proporciona roles para el disenso en cada una de estas etapas.

Consideremos primero el desacuerdo durante la deliberación grupal, antes de la toma de decisiones. Ya vimos que la expresión de desacuerdos durante la deliberación grupal llama la atención de los responsables de la toma de decisiones hacia información distribuida asimétricamente y diversas estrategias de solución de problemas 
que pueden ser relevantes para la solución de los problemas públicos. Tanto el modelo experimentalista de democracia de Dewey como el teorema DSH capturan esta función epistémica del desacuerdo, mientras que el teorema del Jurado de Condorcet la descuida. Además, se necesita la expresión integral de diversos puntos de vista para definir mediante concesiones (Richardson, 1997, pp. 360-363) qué cuenta como un problema de interés genuinamente público en lugar de un interés meramente privado o sectario.

El disenso previo a la toma de decisiones es una condición necesaria para la formación de una voluntad genuinamente colectiva, consistente con la autonomía de cada miembro. Este punto puede parecer sorprendente. Tendemos a pensar que para que un colectivo quiera algo genuinamente, cada unx de sus miembros ya debía quererlo de forma individual y de todo corazón. Gilbert (1989) ha demostrado que la voluntad colectiva no requiere esa unanimidad en el nivel individual, sino simplemente la voluntad de aceptar la decisión colectiva como autorizada para el grupo (incluso si uno disiente en privado), y de hacer su parte en la defensa de la decisión. Westlund (2003) ha demostrado aún más: que la formación de una voluntad colectiva consistente con la autonomía de cada unx de lxs miembros del grupo requiere cierta resistencia en el nivel individual a la propuesta de cualquier otrx, de modo que el objeto eventual de la voluntad conjunta sea el producto de hacer lugar a las demandas de los otros y ceder en las propias y no de una subordinación ciega. La pura deferencia a un líder que afirma encarnar la voluntad colectiva, por muy sincera que sea, es incompatible con la autonomía de lxs miembros individuales. Traducido en términos políticos, el contraste que se dibuja aquí es entre la democracia y las políticas del entusiasmo de masas que nos son familiares desde la Revolución Francesa hasta el fascismo y el comunismo del siglo XX.

Por importante que sea la diversidad epistémica para descubrir la solución de los problemas de interés público, e incluso para construir la definición misma de estos problemas, todavía podemos preguntarnos si tiene alguna función en la etapa de la toma de decisiones propiamente dicha. ¿Por qué permitir que la decisión de una mayoría se mantenga como la decisión de todos? ¿Por qué conformarse con esto, en lugar de la unanimidad? La respuesta convencional es que los grupos difícilmente podrían decidir algo si se requiriera la unanimidad. Pero esta respuesta no es plenamente satisfactoria, dado que algunos grupos -reuniones de cuáquerxs, por ejemplo- sí toman decisiones por consenso.

Una respuesta más satisfactoria considera los costos de lograr el consenso. Precisamente porque las decisiones colectivas son tan a menudo necesarias y urgentes, condicionar las decisiones al logro del consenso a menudo conduce a una presión indebida e incluso a la coerción de las minorías disidentes. Esa presión coercitiva es objetable en sí misma. También conlleva graves costos epistémicos. El consenso implica que todos están de acuerdo en que todas las objeciones a una propuesta han sido satisfechas o al menos superadas por consideraciones más importantes. Por lo tanto, se espera que las partes en un consenso mantengan la calma una vez que se toma una decisión, sobre el supuesto de que han sido contempladas todas sus reservas. Esta norma suprime la difusión pública y la respuesta a las continuas reservas que lxs individuxs puedan tener sobre la decisión. La norma de la mayoría, aunque permite a las mayorías ir por encima de las objeciones de las minorías, no pretende responder plenamente a esas objeciones. La disidencia de la minoría permanece abierta en lugar de ser suprimida, recordándonos que cualquier decisión dada sigue estando asolada por objeciones no resueltas (Manin, 1987, p. 359).

Por esta razón, lxs individuxs deben ser libres de disentir no solo en la etapa de votación, sino después de que se tome una decisión. Esto requiere la institucionalización de una "oposición leal." Sin una oposición que recuerde al público las continuas objeciones a las decisiones colectivas y que plantee alternativas, es imposible la rendición de cuentas de las personas a cargo de la toma de decisiones. Nada las obligaría a reconsiderar sus decisiones. solo con esa oposición continua puede realizarse el falibilismo y la capacidad institucional para la experimentación -la revisión de las propias decisiones sobre la base de la experiencia con sus consecuencias-. Los enfoques epistémicos de la democracia, como el Teorema del Jurado de Condorcet, que representan a la mayoría como prácticamente infalible, no logran explicar la importancia epistémica del disenso posterior a las decisiones. Ese disenso es necesario no solo para mantener a la mayoría bajo control, sino para asegurar que la toma de decisiones sea deliberativa -emprendida con un espíritu experimental- en lugar de simplemente impuesta.

Un poder que no se enfrenta a ningún obstáculo tendrá menos motivos para deliberar sobre sus decisiones y menos necesidad de justificarlas. El verdadero objetivo del pluralismo de las contrafuerzas no es el equilibrio, sino la deliberación misma. (Manin, 1987, p. 361).

Por lo tanto, cualquier evaluación de las capacidades epistémicas de las democracias particulares debe prestar mucha atención a sus instituciones y normas de disenso: ¿hay canales variados, abiertos y accesibles para que personas de todo tipo de recorrido de vida, en todas las posiciones sociales, expresen públicamente su disenso? ¿Las normas sociales acogen con agrado la expresión del disenso de todas las partes descontentas? ¿Exigen que los responsables de la toma de decisiones tomen en serio el disenso, y los responsabilizan si no lo hacen? Las respuestas negativas a estas preguntas indican debilidades epistémicas en la toma política de decisiones. 


\section{Evaluación de las capacidades epistémicas de las instituciones democráticas: estudio de caso de grupos forestales comunitarios en el sur de Asia}

El abordaje experimentalista de Dewey sobre la democracia como ejercicio colectivo de inteligencia práctica ofrece recursos ricos para evaluar las capacidades epistémicas de determinadas instituciones democráticas y para sugerir reformas en pos de la mejora de esos poderes. Supongamos que podemos identificar un problema de interés público. Podemos entonces investigar qué información es relevante para resolver ese problema y quién tiene esa información. Entendiendo esto así, podemos trazar los caminos por los que esta información se transmite a lxs tomadorxs de decisiones políticas - - , si el sistema democrático no funciona bien-, los obstáculos institucionales e informales para transmitir esa información y asegurar que sea efectivamente incorporada por las personas a cargo de la toma de decisiones. Una vez identificados esos obstáculos epistémicos, podemos experimentar con reformas para superarlos, probando las reformas por su impacto final en las capacidades epistémicas de los órganos de toma de decisiones.

La investigación de Bina Agarwal sobre los grupos forestales comunitarios (GFC) en la India y Nepal proporciona un excelente estudio de caso sobre cómo el modelo experimentalista de democracia de Dewey puede dar forma a los esfuerzos para mejorar las capacidades epistémicas de determinadas instituciones democráticas. Los GFC son instituciones democráticas, organizadas al nivel de las aldeas, encargadas de la tarea de gestionar de manera sostenible los bienes comunes forestales. Se crearon en respuesta a la grave degradación de los bosques locales que acompañó a la explotación no regulada de los bienes comunes. En muchas aldeas, antes de que se establecieran los GFC, los bosques degradados proporcionaban poco más que ramitas y hierba de monzón. De cinco a siete años después de que los GFC comenzaran a administrar los bienes comunes, las aldeas disfrutaron de bosques florecientes, mayor biodiversidad y mayores ingresos (Agarwal, 2000, p. 285).

Esto suena como una historia de éxito rotundo. Sin embargo, muchos de estos beneficios de los GFC se obtuvieron a un costo severo para las mujeres, que son las principales usuarias del bosque (Agarwal 2001, pp. 1630-1634). La división generizada del trabajo en el sur de Asia asigna a las mujeres la tarea de recolectar leña para cocinar y calentar el agua de los baños y forraje para los animales de granja. Cerca de la mitad de los GFC encuestados por Agarwal han prohibido la recolección de combustible y forraje de los bosques de las aldeas. Esto ha forzado a las mujeres de las aldeas afectadas a viajar mucho más lejos para obtener estos productos. En muchas aldeas, las mujeres que antes solo necesitaban 1-2 horas diarias para recoger leña ahora necesitan 4-5 horas para hacerlo y deben sacar a sus hijas de la escuela para ayudarlas, limitando así la educación de sus hijas (Agarwal, 2000, p. 286). Como extrañas que entran en aldeas distantes que tienen bosques comunales no regulados, han sufrido abusos de los lugareños hostiles frente a su intrusión. Las mujeres han tenido que recurrir a combustibles alternativos, como el estiércol y las hojas secas, que requieren una atención constante para mantenerse encendidas. Esto ha aumentado el tiempo de cocción y ha impedido que las mujeres se dediquen a otras tareas mientras se cocinan los alimentos. Los combustibles de menor calidad también aumentan la contaminación del aire en los espacios cerrados, en detrimento de la salud de las mujeres, ya que ellas deben pasar más tiempo dentro del hogar que los hombres. Las tierras de cultivo han tenido que desviarse a la producción de combustible y forraje, mientras que se ha tenido que gastar más dinero en el costoso kerosene. Algunas mujeres han tenido que renunciar a quemar combustible para calefacción en el invierno (Agarwal, 2001, p. 1634). Las mujeres también se quejan de que los guardias masculinos asignados a la tarea de hacer cumplir las prohibiciones de recoger leña en el bosque son ineficaces, porque, al no haber tenido que recoger leña ellos mismos, no saben dónde están los mejores terrenos de forraje. Ante la ineptitud de los guardias, que no consiguen impedir que los infractores recojan madera, algunas mujeres han decidido desafiar a los propios guardias.

Así, las mujeres del sur de Asia tienen varias quejas sobre la forma en que los GFC de sus aldeas gestionan los bosques comunes. También sugieren soluciones. Sostienen que los bosques podrían sostener niveles de forrajeo mucho más altos que los permitidos en la actualidad, especialmente si se plantaran más árboles de las especies óptimas para usos domésticos. También sostienen que guardias mujeres o de grupos mixtos serían más eficaces para detener a lxs infractorxs que los guardias exclusivamente varones, porque las mujeres saben mejor dónde es probable que lxs infractorxs busquen madera, no se ven disuadidas por la amenaza de acusaciones de acoso sexual por parte de las mujeres que son detenidas y porque, al ser más eficaces, las mujeres respetarían sus órdenes más que las órdenes de los ineptos guardias exclusivamente varones (Agarwal 2000, pp. 288-289).

Hay razones para creer que las mujeres tienen razón. Debido a que la división generizada del trabajo asigna a las mujeres la responsabilidad de recolectar madera, las mujeres también saben mejor que los hombres cuánta leña se puede recolectar de forma sostenible en el bosque, qué especies son las mejores para combustible y forraje y dónde es probable que lxs recolectorxs vayan a recoger la madera. Este es un caso clásico de conocimiento situado que se distribuye asimétricamente por género. Esto no quiere decir que los hombres no puedan descubrir este conocimiento. De hecho, lxs expertxs en recursos naturales externos -algunos de los cuales, presumiblemente, son hombres- han confirmado la opinión de las mujeres locales de que los bosques comunitarios pueden sostener la recolección de madera a niveles mucho más altos de lo que han permitido 
muchos GFC (Agarwal, 2001, p. 1635). Sin embargo, las aldeas pobres de Asia meridional rara vez tienen acceso a los conocimientos reunidos por lxs expertxs en recursos naturales con entrenamiento formal. Su dependencia de los conocimientos generados localmente hace que sea aún más importante que aprovechen los conocimientos situados de las mujeres para resolver los problemas identificados localmente.

¿Por qué muchos GFC no están haciendo un uso efectivo del conocimiento situado de las mujeres locales? Agarwal identifica numerosos obstáculos formales e informales para la recepción de las ideas de las mujeres por parte de los GFC. La membresía de las mujeres en los GFC suele ser baja: en promedio, menos del 10\% de lxs miembros de los GFC son mujeres (Agarwal, 2001, p. 1626). Las normas que restringen la pertenencia a una persona por hogar junto con la norma sexista de que el "jefe" masculino del hogar representa sus intereses ante el mundo exterior limitan gravemente la participación de las mujeres. La división generizada del trabajo junto con los horarios de las reuniones de los CFG que coinciden con las tareas domésticas de las mujeres, como cocinar y alimentar a los animales, impiden que las mujeres asistan a las reuniones, aunque sean miembros. Las mujeres a menudo no se enteran de las reuniones o decisiones de los GFC y con menos frecuencia aún se les consulta sobre esas decisiones. Los hábitos de los miembros masculinos de beber, ir de juerga y meterse en líos durante las reuniones del CFG funcionan como un disuasivo adicional para la presencia femenina. Otras normas sexistas, que prescriben que las mujeres se sienten separadas de los hombres (en la práctica, en el fondo o en el lateral de la sala, donde rara vez se las reconocerá), que guarden silencio y no desafíen la autoridad de los hombres, impiden que los miembros femeninos se expresen. Cuando algunas juntan el valor de hablar, los hombres, suponiendo que las mujeres no tienen nada serio que aportar, se niegan a escuchar o incluso abandonan la sala (Agarwal, 2001, pp. 1626-1628; 1638-1640).

Antes de que el conocimiento de las mujeres pueda ser usado para resolver los problemas que ellas han identificado, los GFC deben ser reformados para que las mujeres puedan unirse, hablar y ser escuchadas. Lo ideal sería que el orden político estuviera estructurado de tal manera que incluyera métodos de autocorrección, para que pudiera aumentar constantemente sus capacidades epistémicas. Este es el punto del modelo de democracia de Dewey como una encarnación del método científico. Así como la solución a los problemas científicos es hacer más ciencia, "la cura para las dolencias de la democracia es más democracia" (Dewey, 1981c, p. 327). Porque la democracia, como la ciencia, encarna las dos prácticas cruciales para la autocorrección: el disenso y la experimentación.

En algunas aldeas con GFC hostiles a estas demandas, las mujeres han forzado la cuestión de la exclusión femenina en la agenda de los GFC desafiando a los guardias exclusivamente varones y negándose a obedecer las reglas de forrajeo adoptadas por los GFC dominados por los varones que ponen en desventaja a las mujeres. El vigoroso disenso de las mujeres ha obligado a algunos GFC a añadir mujeres a sus patrullas, experimento que ha demostrado su valor por el hecho de que las guardias de mujeres han mejorado la eficacia de las patrullas y la legitimidad de las normas que aplican (Agarwal, 2000, p. 289). Las mujeres también han presentado sus quejas sobre la exclusión ante otras instituciones, como el gobierno nacional y las ONG, que luego presionan a los GFC para que incluyan más mujeres (Agarwal, 2000, p. 304).

El disenso trabaja en conjunto con la experimentación, con el fin de descubrir cómo hacer que el disenso mismo sea más efectivo como aporte a la deliberación. En los GFC de Asia meridional, muchas mujeres han encontrado difícil expresar su desacuerdo porque han internalizado las normas generizadas que indican silencio y deferencia hacia los varones. Les faltaba el valor para hablar al temer no solo que sus opiniones fueran ignoradas por los varones, sino que su reputación se viera dañada por la participación en entornos públicos mixtos. La experimentación ha demostrado que la presencia de una masa crítica de mujeres en las reuniones del GFC reduce la estigmatización de las mujeres que participan. Con una masa crítica, la participación de las mujeres se considera normal, más que una aberración. Las mujeres también han descubierto que toman mayor coraje para hablar en las reuniones del GFC si tienen reuniones previas entre sí. La práctica de hablar en grupos de mujeres les ayuda a encontrar el valor para hablar en grupos mixtos. La discusión en un grupo de mujeres también ayuda a las mujeres a articular una agenda común. Esto aumenta su eficacia en las reuniones del GFC, tanto porque están más dispuestas a hablar, sabiendo que sus puntos serán por lo menos atendidos por otras mujeres, como porque el hecho de que sus puntos sean abordados por otros refuerza su prominencia en todo el grupo, haciéndolos más difíciles de ignorar para los varones (Agarwal, 2001, pp. 1643-1644).

El estudio de Agarwal sobre las GFC ilustra los méritos y defectos relativos de los tres modelos epistémicos de democracia considerados en este artículo. La diversidad de participantes por género, incluidos los conocimientos de las mujeres, desempeña un papel fundamental en el aumento de la capacidad de las políticas de los GFC para resolver el problema de la escasez de leña y forraje. Lo mismo sucede con el debate: las mujeres necesitan hablar entre ellas para elaborar un programa común que puedan llevar a la sala y necesitan dirigirse a todo el grupo para que sus ideas se incorporen a las políticas de los GFC. Dado que el teorema del Jurado de Condorcet no representa la diversidad y el debate como algo fundamental para las capacidades epistémicas de la democracia, no puede ver un problema cuando estos elementos faltan en las instituciones democráticas reales. El Teorema DSH es superior al Teorema del Jurado de Condorcet en su representación de estas características de la democracia (en particular, el valor de la discusión en pequeños grupos para generar propuestas de soluciones a los problemas). Sin embargo, su modelo de democracia es 
incompleto. El estudio de Agarwal muestra cómo la inclusión universal desempeña un papel no instrumental en la legitimación de los resultados: cuando las mujeres fueron excluidas de toda voz en la formulación y ratificación de las normas que restringen la búsqueda de alimento, percibieron esas normas como injustas y era más probable que las infringieran. Su disenso posterior a la decisión en forma de desobediencia civil impulsó el cambio institucional. Esto inspiró a algunos GFC a incorporar los aportes del disenso en el proceso de toma de decisiones, lo que les permitió adoptar decisiones más legítimas y aplicables. El modelo experimentalista de democracia de Dewey es superior al Teorema DSH al representar los valores de inclusión universal y retroalimentación dinámica como esenciales para las capacidades epistémicas de la democracia. El estudio de caso de Agarwal muestra cómo el disenso funciona de forma dinámica, no solo para alterar el contenido de las decisiones democráticas, sino los propios procedimientos de toma de decisiones. Las mujeres se opusieron tanto al contenido como al proceso de las decisiones excluyentes de los GFC y su disenso cambió ambos. El modelo experimental de Dewey captura precisamente esta interacción dinámica de resultado y proceso. La insatisfacción expresada con los resultados provoca la alteración de los procedimientos que conducen a tales decisiones insatisfactorias.

El modelo de democracia de Dewey nos ayuda a ver cómo los experimentos de los GFC vinculados con reformas democráticas pueden ser vistos provechosamente como experimentos en epistemología social aplicada, naturalizada y feminista. Los experimentos de los GFC están diseñados para responder a la pregunta: ¿cómo se pueden reformar las instituciones democráticas para obtener y aprovechar el conocimiento situado de las mujeres, necesario tanto para definir como para resolver problemas de interés público? Las políticas públicas que regulan los bosques comunitarios que se forman sin aprovechar el conocimiento situado de las mujeres son ineficientes - desaprovechan los recursos comunitarios y desarrollan recursos (especies arbóreas) que son menos útiles que otras alternativas para la comunidad en su conjunto-. También son inequitativas, pues distribuyen los beneficios y las cargas en grave desventaja para las mujeres. Estos hechos generan un argumento convincente para que la reforma democrática incluya a las mujeres como participantes en pie de igualdad en el debate. Esa reforma aumenta las capacidades epistémicas de los GFC, haciéndolos más sensibles al conocimiento situado de las mujeres. La mejora epistémica y la reforma democrática van de la mano, tal y como predice el modelo experimentalista de democracia de Dewey. Así como la epistemología naturalizada es la aplicación de la investigación científica para mejorar la investigación misma, la reforma democrática es la aplicación de la epistemología social experimental para mejorar la investigación colectiva en la definición y solución de los problemas públicos.

\section{Referencias bibliográficas}

Agarwal, Bina (2000). Conceptualizing environmental collective action: Why gender matters [Conceptualización de la acción colectiva medioambiental: Por qué importa el género]. Cambridge Journal of Economics, 24, 283-310.

Agarwal, Bina (2001). Participatory exclusions, community forestry, and gender: An analysis for south asia and a conceptual framework [Exclusiones participativas, silvicultura comunitaria y género: Un análisis para el sur de Asia y un marco conceptual]. World Development, 29(10), 1623-48. https://doi.org/10.1016/S0305-750X(01)00066-3

Anderson, Elizabeth (2002). Consumer sovereignty vs. citizens' sovereignty: Some errors in neoclassical welfare economics [Soberanía de lxs consumidorxs frente a soberanía de lxs ciudadanxs: Algunos errores de la economía neoclásica del bienestar]. En H. Pauer-Studer y H. Nagl-Docekal (Eds.), Freiheit, gleichheit und autonomie: Wiener Reihe. Themen der philosophie (pp. 358-388). Verlag Oldenbourg.

Cohen, Joshua (1986). An epistemic conception of democracy [Una concepción epistémica de la democracia]. Ethics, 97(1), 26-38. https://doi.org/10.1086/292815

Condorcet, Marquis de (1995). An essay on the application of analysis to the probability of decisions rendered by a plurality of votes [Un ensayo sobre la aplicación del análisis a la probabilidad de las decisiones tomadas por una pluralidad de votos]. En McLean Iain y A.B. Urken (Eds.), Classics of social choice (pp. 91-112). University of Michigan Press. (Trabajo original publicado en 1785).

Dewey, John (1976). Valuation and experimental knowledge [Valoración y conocimiento experimental]. En J. A. Boydston (Ed.), The middle works of John Dewey, 1899-1924, (pp. 3-28). Southern Illinois University Press.

Dewey, John (1981a). Creative democracy: The task before Us [Democracia creativa: La tarea que tenemos por delante ]. En J. A. Boydston (Ed.), The later works of John Dewey, 1925-1953 (pp. 224-30). Southern Illinois University Press.

Dewey, John (1981b). Freedom and culture [Libertad y cultura]. En J. A. Boydston (Ed.), The later works of John Dewey, 1925-1953 (pp. 65-188). Southern Illinois University Press.

Dewey, John (1981c). The public and its problems [El público y sus problemas]. En J. A. Boydston (Ed.), The later works of John Dewey, 1925-1953 (pp. 235-372). Southern Illinois University Press.

Estlund, David (1994). Opinion leaders, independence, and Condorcet's Jury theorem [Líderes de opinión, independencia y el teorema del jurado de Condorcet]. Theory and Decision, 36(2), 131-62. https://doi.org/10.1007/BF01079210 
Estlund, David (1997). Beyond fairness and deliberation: The epistemic dimension of democratic authority [Más allá de la equidad y la deliberación: La dimensión epistémica de la autoridad democrática]. En J. Bohman y W. Rehg (Eds), Deliberative democracy (pp. 173-204). MIT Press.

Fey, Mark (2003). A note on the Condorcet jury theorem with supermajority voting rules [Una nota sobre el teorema del jurado de Condorcet con reglas de votación por supermayoría]. Social Choice and Welfare, 20(1), 27-32. https://doi. org/10.1007/s003550200157

Gaus, Gerald (1997). Does democracy reveal the voice of the people? Four takes on Rousseau [¿La democracia revela la voz del pueblo? Cuatro opiniones sobre Rousseau]. Australasian Journal of Philosophy, 75(2), 141-62. https://doi. org/10.1080/00048409712347761

Gilbert, Margaret (1989). On social facts [Sobre los hechos sociales]. Princeton University Press. https://doi. org/10.1515/9780691214627

Grofman, Bernard y Feld, Scott (1988). Rousseau's general will: A condorcetian perspective [La voluntad general de Rousseau: Una perspectiva condorcetiana]. American Political Science Review, 82(2), 567-76. https://doi. org/10.2307/1957401

Hayek, Friedrich (1945). The use of knowledge in society [El uso del conocimiento en sociedad]. American Economic Review, 35: 519-30.

Herzog, Don (2000). Externalities and other parasites [Externalidades y otros parásitos]. University of Chicago Law Review, 67, 895-923. https://doi.org/10.2307/1600344

Hong, Lu y Page, Scott (2004). Groups of diverse problem solvers can outperform groups of high-ability problem solvers [Los grupos de diversxs solucionadorxs de problemas pueden superar a los grupos de solucionadorxs de problemas de alta capacidad]. Proceedings of the National Academy of Sciences, 101, 16385-89. https://doi.org/10.1073/ pnas.0403723101

List, Christian y Goodin, Robert (2001). Epistemic democracy: Generalizing the Condorcet jury theorem [La democracia epistémica: Generalización del teorema del jurado de Condorcet]. Journal of Political Philosophy, 9(3), $277-306$. https://doi.org/10.1111/1467-9760.00128

Manin, Bernard (1987). On legitimacy and political deliberation [Sobre la legitimidad y la deliberación política]. Political Theory, 15, 338-68. https://doi.org/10.1177/0090591787015003005

Page, Scott (2007). The difference: How the power of diversity creates better groups, firms, schools, and societies [La diferencia: Cómo el poder de la diversidad crea mejores grupos, empresas, escuelas y sociedades]. Princeton University. https://doi.org/10.2307/j.ctt7sp9c

Putnam, Hilary (1990). A reconsideration of deweyan democracy [Una reconsideración de la democracia deweyana]. Southern California Law Review, 63, 1671-97. https://doi.org/10.1515/9781400838684-023

Richardson, Henry (1997). Democratic intentions [Intenciones democráticas]. En J. Bohman y W. Rehg, Deliberative democracy (pp. 349-82). MIT Press. https://doi.org/10.7551/mitpress/2324.003.0015

Surowiecki, James (2004). The wisdom of crowds: Why the many are smarter than the few and how collective wisdom shapes business, economies, societies, and nations [La sabiduría de las multitudes: Por qué lxs muchxs son más inteligentes que lxs pocxs y cómo la sabiduría colectiva da forma a los negocios, las economías, las sociedades y las naciones]. Doubleday.

Westlund, Andrea (2003). Selflessness and responsibility for self: Is deference compatible with autonomy? [Desinterés y responsabilidad por unx mismx: ¿Es compatible la deferencia con la autonomía?]. Philosophical Review, 112(4), 483523. https://doi.org/10.1215/00318108-112-4-483 